\title{
OPEN The Human Lung Glycome Reveals Novel Glycan Ligands for Influenza A Virus
}

\author{
Nan Jia $\mathbb{1}^{1,4}$, Lauren Byrd-Leotis $\mathbb{1}^{1,3,4}$, Yasuyuki Matsumoto ${ }^{1}$, Chao Gao ${ }^{1,3}$, \\ Alexander N. Wein ${ }^{2}$, Jenna L. Lobby ${ }^{2}$, Jacob E. Kohlmeier $\mathbb{B}^{2}$, David A. Steinhauer $\mathbb{B}^{2,3^{*}}$ \& \\ Richard D. Cummings $\mathbb{D}^{1,3^{*}}$
}

Glycans within human lungs are recognized by many pathogens such as influenza $A$ virus (IAV), yet little is known about their structures. Here we present the first analysis of the $\mathrm{N}$ - and $\mathrm{O}$ - and glycosphingolipid-glycans from total human lungs, along with histological analyses of IAV binding. The $\mathrm{N}$-glycome of human lung contains extremely large complex-type $\mathrm{N}$-glycans with linear poly- $\mathrm{N}$ acetyllactosamine (PL) [-3Gal $31-4 \mathrm{GlcNAc} \beta 1-]_{n}$ extensions, which are predominantly terminated in $\alpha 2,3$-linked sialic acid. By contrast, smaller $\mathrm{N}$-glycans lack PL and are enriched in $\alpha 2,6$-linked sialic acids. In addition, we observed large glycosphingolipid (GSL)-glycans, which also consists of linear PL, terminating in mainly $\alpha 2,3$-linked sialic acid. Histological staining revealed that IAV binds to sialylated and non-sialylated glycans and binding is not concordant with respect to binding by sialic acid-specific lectins. These results extend our understanding of the types of glycans that may serve as binding sites for human lung pathogens.

Glycosylation represents the major post-translational modification of cellular proteins ${ }^{1}$, and glycoproteins along with glycolipids are abundantly displayed on the plasma membranes of cells and in secretions ${ }^{2}$. Complex glycoconjugates regulate numerous biological processes such as quality control of protein folding, modulation of gene transcription, cellular signaling and adhesion, and trafficking of all immune cells and platelets ${ }^{3-5}$. The diversity of different human glycans represents the total human glycome, which is vast and undefined, yet anticipated to represent many hundreds of thousands of different structures ${ }^{6}$. Each glycan structure is genetically dictated through specific gene expression of so-called 'glycogenes', which overall number over 500 in the human genome ${ }^{7}$, representing 1-2\% of the genome. However, much of our knowledge of the human glycome is confined to free glycans and those expressed on blood cells and plasma glycoproteins and antibodies ${ }^{8-11}$. While studies on glycomes of individual cell types, cultured cells and human surgical biopsies ${ }^{12-15}$ have advanced our understanding of the potential glycans in the human glycome, few studies have globally analyzed total human tissues or organs.

It is important to define human glycans not only for their roles in systems biology, but to also discern the potential attachment sites and ligands for many infectious organisms and viruses. Human lungs are frequently subjected to infection by a variety of pathogens including viruses and bacteria ${ }^{16-18}$. Influenza A virus (IAV) receptor recognition is one of the most intensively studied examples of pathogen-host interaction, as glycans expressed on the surface of human airway are essential in shaping the initiation of infection by IAV. It has been commonly believed that the IAV envelope protein, hemagglutinin, recognizes glycans with a terminal sialic acid in either $\alpha 2,3$ - or $\alpha 2,6$-linkage and such recognition is required for infection. As a general paradigm, $\alpha 2,6$-sialylated structures are preferentially recognized by human IAVs, whereas avian strains preferentially bind $\alpha 2,3$-sialylated glycans ${ }^{19,20}$

Despite much effort to understand the initial virus-host recognition event, little is known about the natural repertoire of glycans present in the human respiratory system. Current understanding of the human lung glycome remains at the tissue level with limited information obtained from lectin staining and mass spectrometric analyses of surgical biopsies from human lungs ${ }^{21-23}$. It is important to understand the landscape of glycosylation

${ }^{1}$ Beth Israel Deaconess Medical Center, Department of Surgery and Harvard Medical School Center for Glycoscience, Harvard Medical School, Boston, MA, USA. ${ }^{2}$ Department of Microbiology and Immunology, Emory University School of Medicine, Atlanta, GA, USA. ${ }^{3}$ Emory-UGA Center of Excellence of Influenza Research and Surveillance, (CEIRS), Atlanta, GA, USA. ${ }^{4}$ These authors contributed equally: Nan Jia and Lauren Byrd-Leotis. *email: dsteinh@emory.edu; rcummin1@bidmc.harvard.edu 
a

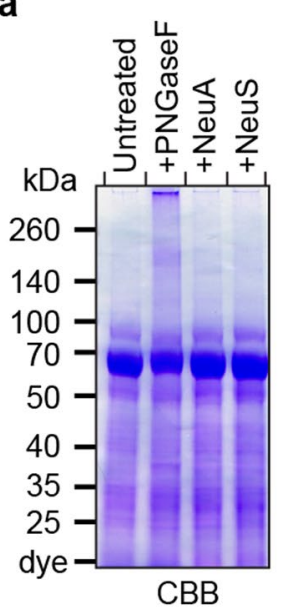

e

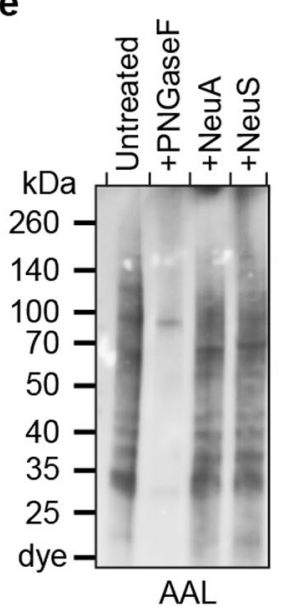

b

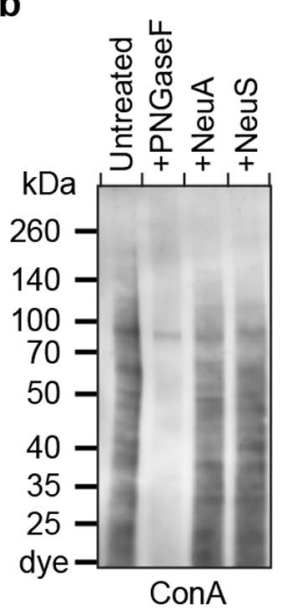

$\mathbf{f}$

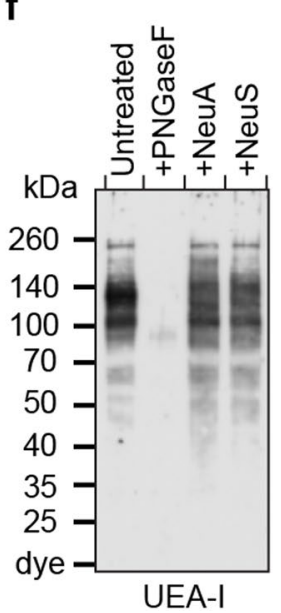

C

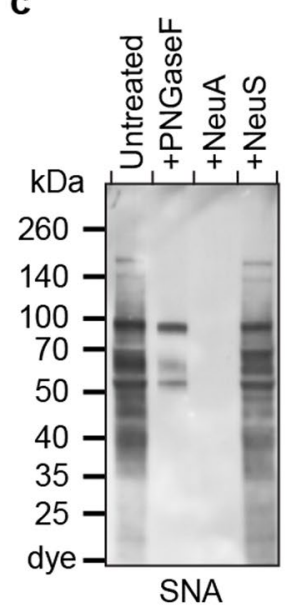

g

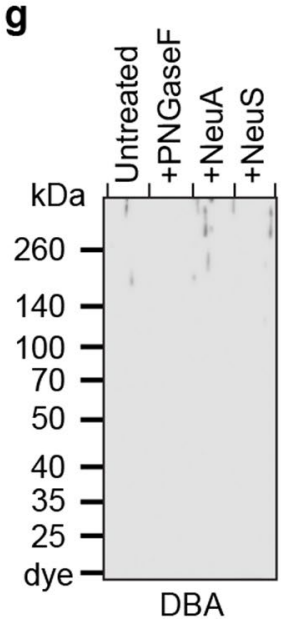

d

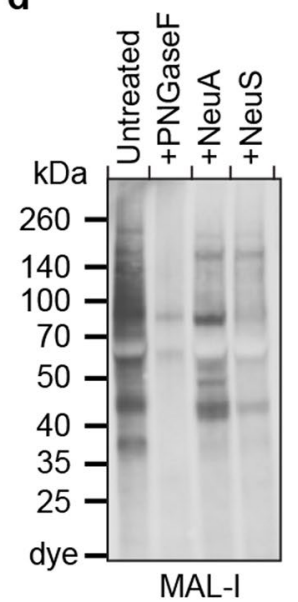

h

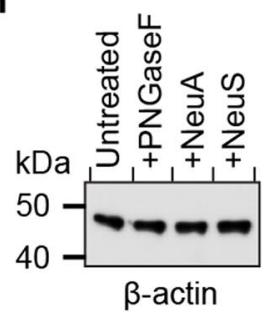

Figure 1. Western blot of human lung. Tissue homogenate in a human lung were treated with PNGase F (PNGaseF), Neuraminidase A (NeuA), or Neuraminidase S (NeuS), and separated by SDS-PAGE. The gel was stained with Coomassie Brilliant Blue solution (a), or analyzed by Western blot using ConA (b), SNA (c), MAL-I (d), AAL (e), UEA-I (f), and DBA (g). $\beta$-actin antibody staining was used as an internal control (h).

at the organ level, as an organ represents a full collection of all pulmonary cell types whereas biopsy specimens only focus on a small area of lung parenchyma, which may not contain all glycan structures found in a lung. Our recent studies using shotgun glycomics of total $\mathrm{N}$-glycans of a human lung challenge the paradigm of sialic acid recognition, as we discovered many IAVs recognize phosphorylated glycans ${ }^{24}$. Thus, it is even more important to gain a fuller understanding of the complexity of the human lung glycome to assess the structures and functions of glycans in lung biology.

Here we present our analyses of the human lung glycome using mass spectrometry (MS), with complementary information generated by Western blot and histochemistry staining with anti-glycan antibodies, lectins and IAVs. We focused on the analysis of $\mathrm{N}$ - and O-glycans released from glycoproteins as well as glycosphingolipid (GSL)-derived glycans that were extracted from a perfused lung of a healthy young adult, the results of which are compared to a lung from a second donor. To advance our understanding of minor glycan species, we established a method to characterize phosphorylated glycans. The present work provides a comprehensive mapping of the human lung glycome and represents the first of its kind to dissect the human glycome at the organ level. This work represents a major step in the goals of the Human Glycome Project, which aims to define the structures and functions of human glycoconjugates (www.human-glycome.org).

\section{Results}

Western blot reveals the expression of sialylated and fucosylated glycans in human

lung. Human lungs used for analyses were perfused upon collection and devoid of blood cells and soluble mucus. We interrogated the glycome of human lung glycoproteins by probing for specific glycan epitopes in a lung homogenate using lectins and antibodies (Fig. 1a-h; Supplementary Fig. 1a-f). Staining with the lectin concanavalin $\mathrm{A}(\mathrm{Con} \mathrm{A})^{25}$ indicated the presence of oligomannose-type, hybrid-type and bi-antennary complex $\mathrm{N}$-glycans in total material. PNGase $\mathrm{F}$ treatment, which specifically removes $\mathrm{N}$-glycans, reduced ConA staining, confirming that human lung contains a variety of $\mathrm{N}$-linked glycans (Fig. 1b). The presence of sialic acid 
a

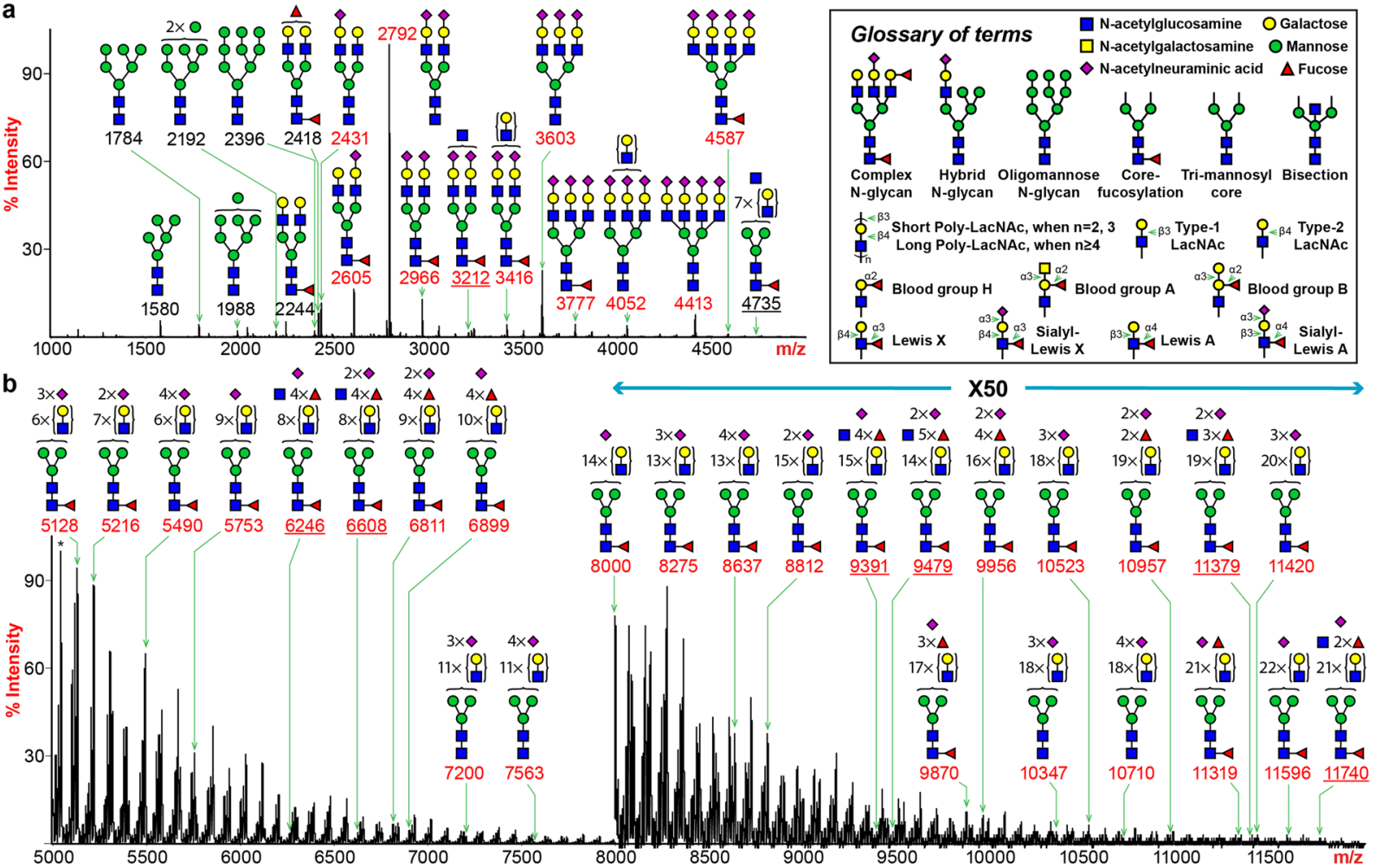

Figure 2. N-glycan MS profile of human lung. All molecular ions detected represent permethylated species and are present in the form of $[\mathrm{M}+\mathrm{Na}]^{+}$. (a) MALDI-TOF-MS spectrum covers the $\mathrm{m} / \mathrm{z}$ range between 1000 and 5000. (b) MALDI-TOF-MS spectrum covers the $\mathrm{m} / \mathrm{z}$ range between 5000 and 12000 . The relative intensity of the most abundant peak in each spectrum is set at $100 \%$. The relative intensity of the highest peak in the panel (b), as indicated by an asterisk (*), equals $0.1 \%$ of the highest peak in panel (a), if both peaks are plotted in the same panel. Selected peak masses were annotated to reflect the diversity of structural features including sialylated glycans (red), non-sialylated (black) and bisected (underline) glycans. Cartoon symbols appear above a curly parenthesis indicates that the sequences corresponding to these compositions cannot be unequivocally defined with linkage information. Symbol representations of monosaccharides are: yellow circle, Galactose; green circle, Mannose; red triangle, Fucose; blue square, N-acetylglucosamine; purple diamond, $\mathrm{N}$-acetylneuraminic acid.

was supported by staining with two commonly used sialic acid-recognizing lectins, Sambucus nigra agglutinin (SNA) and Maackia amurensis lectin-I (MAL-I) ${ }^{26,27}$. SNA, which detects the expression of $\alpha 2,6$-linked sialic acid, bound a wide range of proteins and the staining was not affected by neuraminidase $S$ digestion, which hydrolyses $\alpha 2,3$-linked sialic acids (Fig. 1c). PNGase F partially reduced SNA binding, as $\alpha 2,6$-sialylation is also present on $\mathrm{O}$-glycans. The expression of $\alpha 2,3$-sialylated glycans was assessed by the recognition with MAL-I. Treatment with PNGase F diminished staining by MAL-I, indicating that $\alpha 2,3$-sialylation predominantly occurs on N-glycans (Fig. 1d). Digestion with neuraminidase S significantly diminished staining, although neuraminidase A, which removes sialic acids in all linkages, did not result in complete elimination of MAL-I binding. This result with MAL-I is consistent with our recent finding that MAL-I can recognize some types of non-sialylated complex-type $\mathrm{N}$-glycans ${ }^{28}$.

The expression of fucosylated glycans was detected by Aleuria aurantia lectin (AAL), which preferentially binds glycans containing $\alpha 2-, \alpha 3-, \alpha 4$ - or $\alpha 6$-fucosylated sequences ${ }^{29}$. Fucosylation is predominantly present on N-glycans as PNGase F reduced almost all AAL binding (Fig. 1e). Because fucosylation is associated with the expression of the ABO and the Lewis blood groups, we further differentiated the expression of these glycan epitopes. Positive staining with Ulex europaeus-I (UEA-I), which binds the $\alpha 2$-fucosylated H-type 2 blood group ${ }^{30}$, showed exclusive expression of this modification on N-glycans (Fig. 1f). Staining with Dolichos biflorus agglutinin (DBA) ${ }^{31}$ indicated the absence of the blood group A antigen (Fig. 1g). The Lewis A (LeA) and sialyl-Lewis A (SLeA) antigens were exclusively detected on O-glycans, and not affected by PNGase F treatment, whereas the Lewis X (LeX) epitopes was mainly present on N-glycans, as PNGase F removed staining (Supplementary Fig. 1).

Human lung expresses structurally diverse $\mathbf{N}$-glycans. To elucidate in-depth structural details of the human lung glycome, we performed mass spectrometric characterization on major glycan classes. The MALDI-TOF MS spectrum of human lung N-linked glycans released by PNGase F revealed striking structural variations up to $\mathrm{m} / \mathrm{z} 12000$ with more than 500 assignable peaks detected (Fig. 2; Supplementary Table 1). The overall profile was dominated by the peak at $\mathrm{m} / \mathrm{z} 2792$, which represented a bi-antennary, di-sialylated structure. 
A full set of oligomannose structures (m/z 1580, 1784, 1988, 2192 and 2396) was detected in the low mass region. Molecular ions consistent with a series of bi-, tri- and tetra-antennary complex-type glycans were observed to carry variable lengths of $\mathrm{N}$-acetyllactosamine (LacNAc) repeats. The highest number of LacNAc units observed from our analysis was (LacNAc) ${ }_{22}$ (e.g., $\mathrm{m} / \mathrm{z}$ 11596). Elongation of LacNAc units occurred exclusively in the linear form as we did not detect the presence of 3,6-subtituted Gal by GC-MS linkage analysis, which corresponds to branched PL chains (Supplementary Table 2). Mono-, di-, tri- and tetra-sialylation were observed and predominantly occurred in the form of Neu5Ac-LacNAc on the non-reducing ends. A minor form of sialylation was also detected where the sialic acid was linked to the GlcNAc (Supplementary Fig. 2). A significant proportion of complex type N-glycans was mono-fucosylated (e.g., m/z 2605, 2966, 3416 and 3777), a structural feature proposed to be core fucosylation with an $\alpha 6$-linkage and was confirmed with GC-MS linkage analysis (Supplementary Table 2; Fig. 2). In the higher $\mathrm{m} / \mathrm{z}$ regions, the compositions of a series of complex glycans were consistent with multi-fucosylated structures with sialylation (e.g., $\mathrm{m} / \mathrm{z} 6811,6899,9870$ and 9956 ). Detection of those peaks indicated the expression of blood group $\mathrm{H}, \mathrm{LeA} / \mathrm{X}$ and SLeA/X antigens and was confirmed by tandem MS and GC-MS analyses (Supplementary Table 2; Fig. 2b,c). Molecular ions corresponding to structures with an additional HexNAc were observed and confirmed to be a bisecting GlcNAc by GC-MS experiment (Supplementary Table 2; Fig. 2a, e.g., m/z 3212, 4735, 6246, 6608, 9391 and 9479).

By comparing the relative intensities of peaks from the MALDI-TOF spectrum, we could dissect the patterns of human lung $\mathrm{N}$-glycosylation in a semi-quantitative manner (Supplementary Fig. 2d). Sialylation represents a major modification as $\sim 75 \%$ of the $\mathrm{N}$-glycans carried at least one sialic acid. Of the sialylated species, $\sim 60 \%$ of glycans were modified with two Neu5Ac residues. The proportions for tri- and tetra-sialylated structures were relatively minor. They together constituted about $10 \%$ of the total sialylated glycans. Although $\sim 500$ fucosylated species were identified from our analysis, in terms of relative quantities, only $\sim 30 \%$ of the glycans were fucosylated. Approximately $80 \%$ of the fucosylated glycans displayed a single fucose. The proportion of glycans with a second fucose dropped sharply to $15 \%$ while the occurrences of tri-, tetra- and penta-fucosylation were dramatically lower. Nonetheless, a few structures with six (e.g., $\mathrm{m} / \mathrm{z} 4823,5185,5273,5722,6083$ and 6171 ) or seven (e.g., $\mathrm{m} / \mathrm{z} 5447$ ) fucose residues were detected with extremely low abundances. Lastly, we looked at the proportions of bisected glycans. Approximately $5 \%$ of the human lung N-glycans carried a bisecting GlcNAc. In contrast to the non-bisected glycans, sialylation was not a prominent terminal modification as only $25 \%$ of bisected glycans were sialylated. Conversely, most of bisected structures were fucosylated (80\%).

Human lung expresses both $\alpha 2,3$ - and $\alpha 2,6$-sialylated $\mathrm{N}$-glycans. We further explored the linkage of sialic acids by treatment with neuraminidases A and S (Fig. 3; Supplementary Fig. 3). Digestion of human lung $\mathrm{N}$-glycans with neuraminidase A resulted in complete de-sialylation (Fig. 3b; Supplementary Fig. 3b). The highest peak with the $\mathrm{m} / \mathrm{z}$ at 2070 represented a bi-antennary structure without core fucosylation. The dramatic increase of this reaction product was consistent with the dominant expressions of its corresponding sialylated structures prior to the digestion (Fig. 3a, m/z 2431 and 2792). The compositions of the six most abundant molecular ions were consistent with fucosylated or non-fucosylated tri-mannosyl core structures carrying (LacNAc) ${ }_{2-4}$ repeats $(\mathrm{m} / \mathrm{z} 2070,2244,2519,2693,2968$ and 3142). As backbones for the addition of sialic acids, they constituted $\sim 80 \%$ of the human lung $\mathrm{N}$-glycome. Further extensions with LacNAc units led to the expression of glycans displaying PL motifs where the highest detectable number of repeats was (LacNAc) ${ }_{23}$ (Supplementary Fig. 3b, m/z 11684). A second series of backbone structures for sialylation included glycans carrying a bisecting GlcNAc (Fig. 3b, $\mathrm{m} / \mathrm{z} 2489,2938,3388$ and 3837). Although bisected glycans were estimated to make up as low as $5 \%$ of the total $\mathrm{N}$-glycome, they can be elongated equally well with the highest detectable number of LacNAc repeat being (LacNAc) ${ }_{22}$ (Supplementary Fig. 3b, m/z 11481).

Compared to the profile of untreated lung, the bi-antennary, di-sialylated structure at $\mathrm{m} / \mathrm{z} 2792$ remained the most abundant glycan after the enzymatic treatment, indicating both sialic acids were mostly $\alpha 2,6$-linked (Fig. 3a,c). However, a small proportion of this structure also possesses $\alpha 2,3$-sialylation, as an increase in the relative intensity of its partially de-sialylated product at $\mathrm{m} / \mathrm{z} 2431$ was detected after the reaction. Other noticeable changes in the lower end of the spectrum included the emergence of the de-sialylated structures (e.g., m/z 2244, 2693,3142 and 3241) due to partial or complete removal of sialic acids from their sialylated counterparts prior to the neuraminidase S digest (Fig. 3a; e.g., m/z 2966, 3602 and 3777). In the higher mass regions, the MALDI-TOF spectrum after neuraminidase $S$ was more comparable to that of the neuraminidase A treated glycome than the control (Fig. 3a-c; m/z 5000-7000). This was exemplified by the appearance of prominent non-sialylated peaks, all of which were detected after the enzymatic digest by either of the enzyme (e.g., $\mathrm{m} / \mathrm{z} 5013,5114,5185$, $5389,5838,6287$ and 6736). All the major sialylated species after neuraminidase $S$ treatment corresponded to mono-sialylated glycans with $\alpha 2$,6-linkages (e.g., $\mathrm{m} / \mathrm{z}$ 5026, 5098, 5200, 5300 and 5373). Patterns in the high mass regions demonstrated additional degrees of similarities between the profiles of glycans following neuraminidase A or S treatment (Supplementary Fig. 3a-c; m/z $>7000$ ). The compositions of the major identified peaks were consistent with fully desialylated glycans carrying PL extensions (e.g., m/z 7190, 8089, 8988, 9887, 10786 and 11684). They were all detected with similar relative intensities after either neuraminidase A or S digestion. These results demonstrated that large extended $\mathrm{N}$-glycans are mainly $\alpha 2,3$-sialylated. Some minor sialylated species with $\alpha 2,6$-sialylation were detectable and they all represented mono-sialylated structures with various degrees of fucosylation (e.g., $\mathrm{m} / \mathrm{z} 7102,7276,7624,7725,8001,8175$ and 8450 ).

Since the short glycans were significantly more abundant than elongated glycans, we concluded that the human lung $\mathrm{N}$-glycome contained a higher overall proportion of $\alpha 2,6$-sialylation, and these are mainly on shorter complex-type $\mathrm{N}$-glycans, compared to $\alpha 2,3$-sialylated structures that are enriched on larger PL-containing complex-type N-glycans. The observation was also confirmed by GC-MS linkage analysis as a higher relative abundance was detected for the 6-substituted Gal than the 3-subsitituted Gal (Supplementary Table 2). 
a No treatment

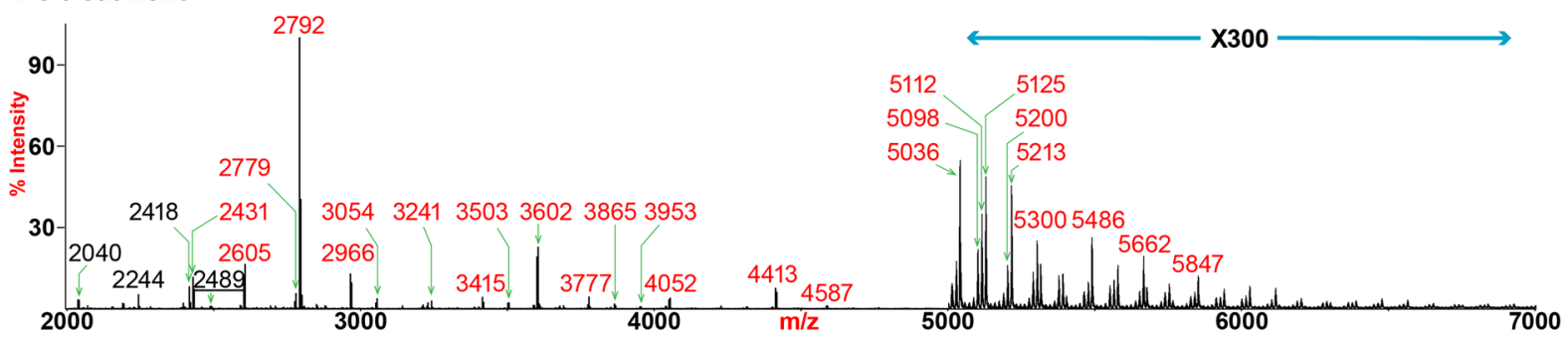

b Treatment with neuraminidase A ( $\alpha 2,3 / 6 / 8 / 9$-linkage specific)

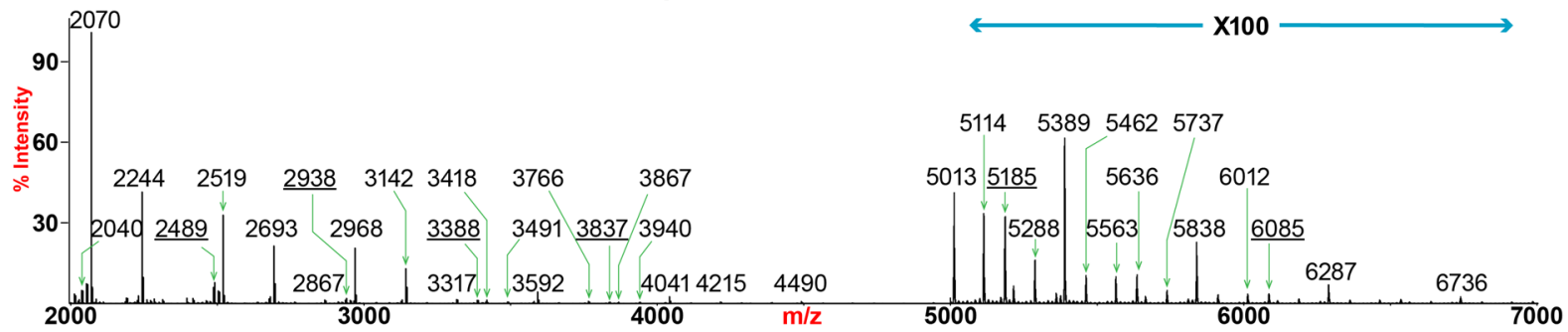

c Treatment with neuraminidase S ( $\alpha 2,3$-linkage specific)

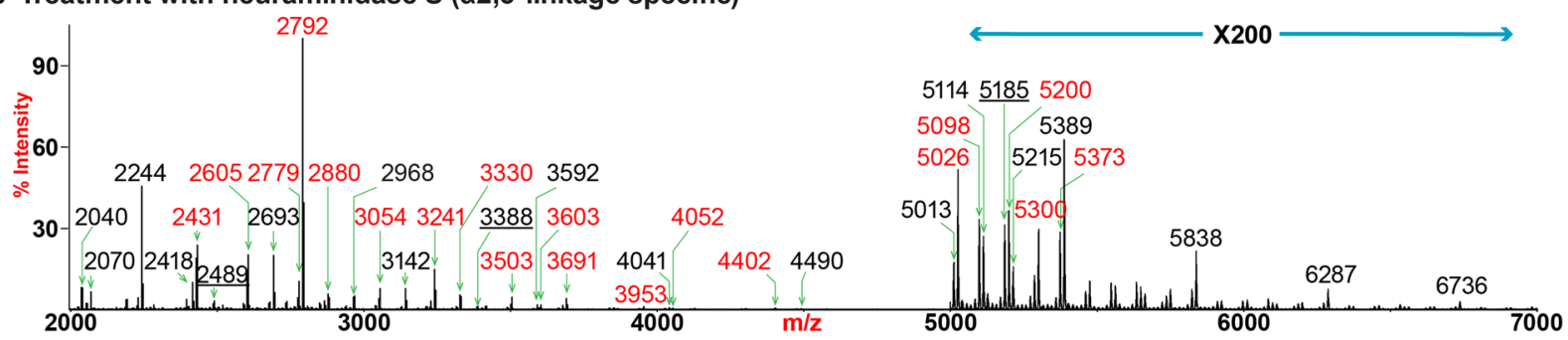

Figure 3. N-glycan MS profiles of human lung following neuraminidase treatment. All molecular ions detected represent permethylated species and are present in the form of $[\mathrm{M}+\mathrm{Na}]^{+}$. (a) MALDI-TOF-MS spectrum of untreated human lung N-glycans. (b) MALDI-TOF-MS spectrum of human lung N-glycans treated with neuraminidase A. (c) MALDI-TOF-MS spectrum of human lung N-glycans treated with neuraminidase $\mathrm{S}$. Peaks representing sialylated glycans are colored in red, non-sialylated glycans are in black and bisected glycans are underlined.

Phosphorylated glycans are minor components of the human lung N-glycome. Our recent studies indicated that IAV can bind to some phosphorylated oligomannose-type N-glycans from the human lung ${ }^{24}$. To characterize such glycans, we isolated the phosphorylated glycans from a human lung by treating lung glycopeptides with Endo H, which releases oligomannose- and hybrid-type N-glycans (Supplementary Fig. 4a). The

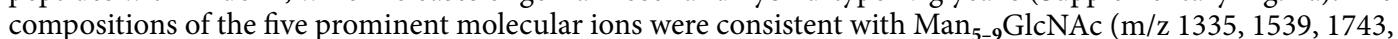
1947 and 2151). Other detectable peaks represented neutral hybrid (e.g., m/z 1580, 1784, 1825, 2029 and 2233) and sialylated hybrid glycans (e.g., m/z 2390, 2595 and 2840). One extremely minor phosphorylated structure was identified with the composition of $\mathrm{Man}_{6}$ GlcNAc-phosphate $(\mathrm{m} / \mathrm{z}$ 1641). We further enriched the phosphorylated species by desialylating the Endo $\mathrm{H}$ released $\mathrm{N}$-glycans and separating via anion exchange chromatography (Supplementary Fig. 4b). We detected a series of phosphorylated oligomannose-type glycans (m/z 1641, $1845,1947,2049,2151$ and 2253) as well as phosphorylated hybrid-type structures (m/z 1886, 2090, 2192, 2395 and 2845). The expression of phosphorylated glycans was confirmed by MS/MS experiments (Supplementary Fig. 4c,d), as the fragment ion at $\mathrm{m} / \mathrm{z} 156$ specifically correspond to a partially permethylated phosphate group $\left(\left[\mathrm{NaPO}^{4}-\mathrm{CH}_{3}+\mathrm{Na}\right]^{+}\right)$rather than a potential sulfate group $\left(\left[\mathrm{NaSO}^{4}+\mathrm{Na}\right]^{+}\right)$.

The O-glycome of human lung mainly expresses two sialylated structures. The soluble mucins of the lung were removed by perfusion originally, and unavailable for analyses here, but we have previously analyzed mucins from normal donors and those with cystic fibrosis ${ }^{32}$. The major residual O-glycome was dominated by the expression of mono- and di-sialylated core-1 structures (Fig. 4a, m/z 896 and 1257; Supplementary Table 3). Molecular ions corresponding to structures with further addition of LacNAc units on the core disaccharide were observed to display sialic acids (m/z 1345, 1706 and 1794). Two fucosylated species (m/z 1519 and 1968) were detected, which were likely to carry the LeA and SLeA antigens, as indicated by the Western blot results and confirmed with MS/MS analysis (Supplementary Figs. 1 and 5a). A minor molecular ion which represented a tri-sialylated structure was detected (Fig. $4 \mathrm{a}, \mathrm{m} / \mathrm{z} 1618$ ). MS/MS analysis indicated that the third sialic acid was added to the Neu5Ac on the 6-arm of the core GalNAc, forming a di-sialyl motif via a potential Neu5Aco2,8-Neu5Ac linkage (Supplementary Fig. 5b).

Neuraminidase A treatment fully removed sialic acids from O-linked glycans (Fig. 4b). A significant elevation in the relative intensity of the core di-saccharide $(\mathrm{m} / \mathrm{z} 534)$ was due to complete de-sialylation of the 
a No treatment

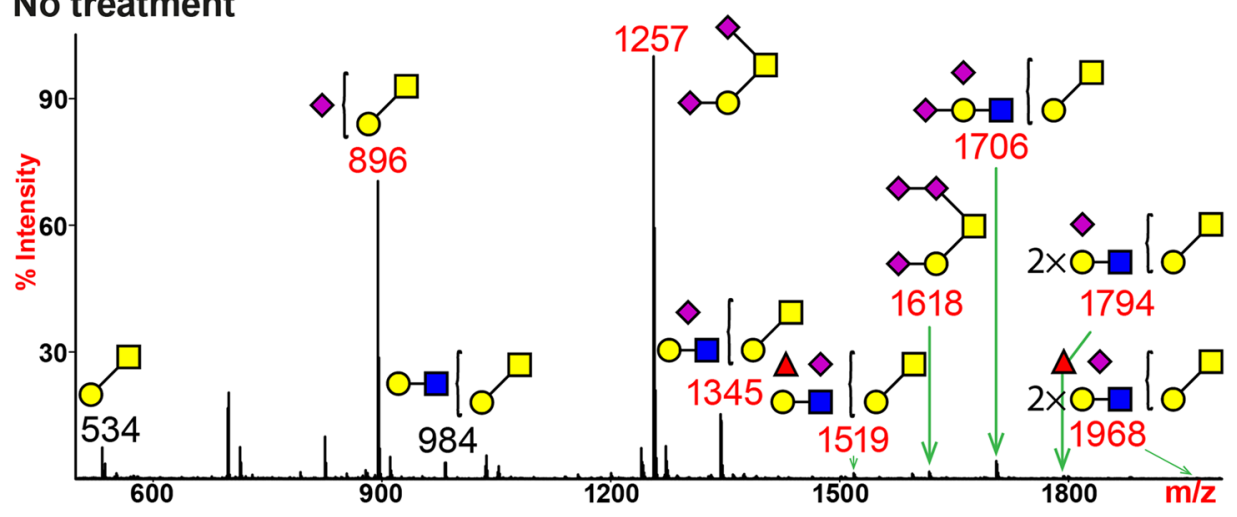

b Treatment with neuraminidase A ( $\alpha 2,3 / 6 / 8 / 9$-linkage specific)

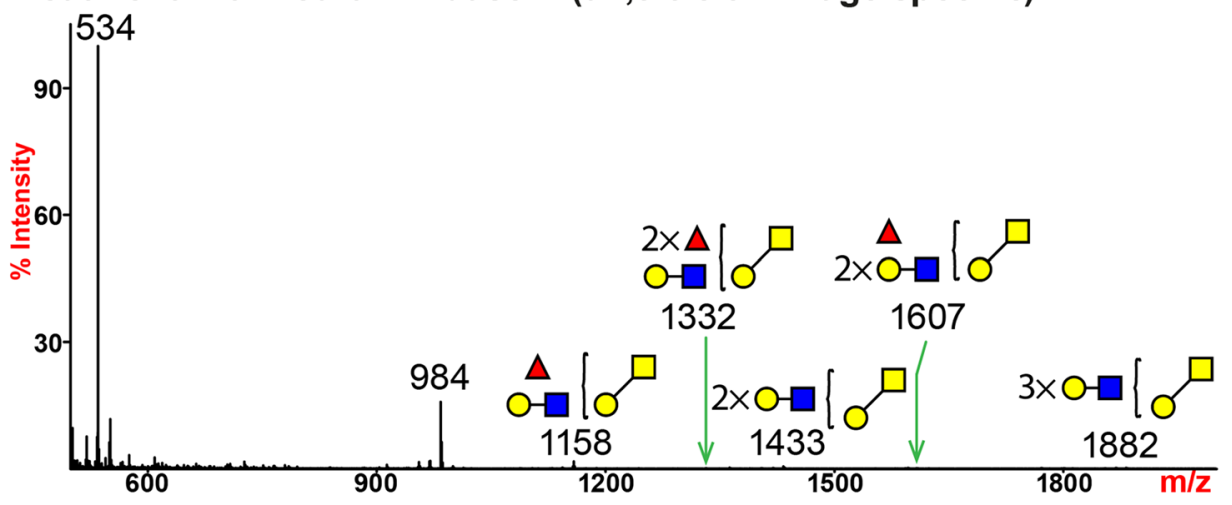

c Treatment with neuraminidase $S$ ( $\alpha 2,3$-linkage specific)

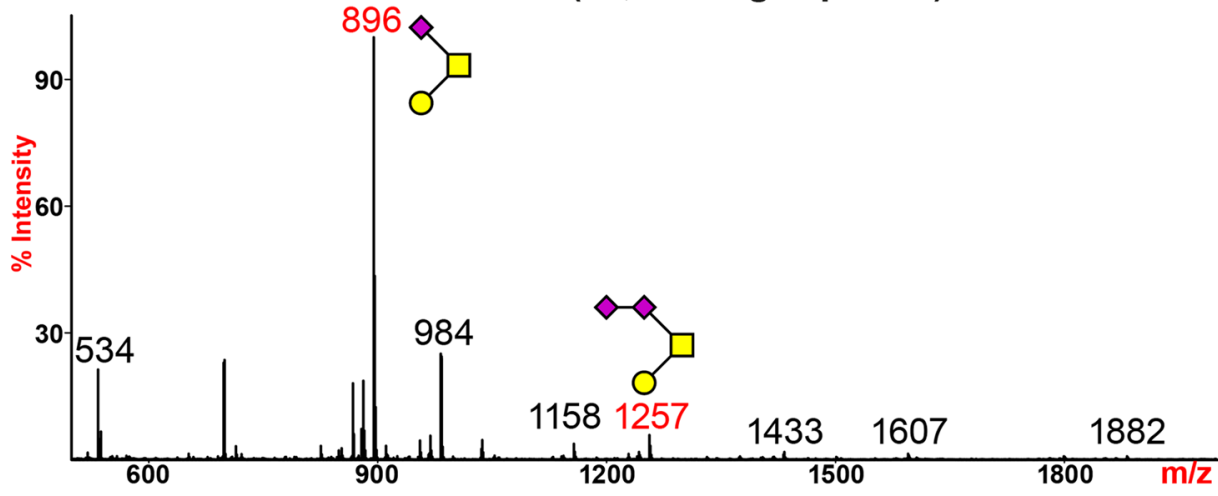

Figure 4. O-glycan MS profiles of human lung following neuraminidase treatment. All molecular ions detected represent permethylated species and are present in the form of $[\mathrm{M}+\mathrm{Na}]^{+}$. (a) MALDI-TOF-MS spectrum of untreated human lung O-glycans. (b) MALDI-TOF-MS spectrum of human lung O-glycans treated with neuraminidase A. (c) MALDI-TOF-MS spectrum of human lung O-glycans treated with neuraminidase S. Peaks representing sialylated glycans are colored in red and non-sialylated glycans are in black. Symbol representations of monosaccharides are: yellow circle, Galactose; green circle, Mannose; red triangle, Fucose; blue square, $\mathrm{N}$-acetylglucosamine; yellow square, $\mathrm{N}$-acetylgalactosamine; purple diamond, $\mathrm{N}$-acetylneuraminic acid.

most abundant mono- and di-sialylated structures (m/z 896 and 1257). One minor structure with two fucoses $(\mathrm{m} / \mathrm{z} 1332)$ was detected after de-sialylation. A heterogeneous expression of $\alpha 2,3$-and $\alpha 2,6$-linkages of sialic acids was revealed by neuraminidase $S$ treatment (Fig. 4c). A significant reduction in the relative intensity of the di-sialylated core structure $(\mathrm{m} / \mathrm{z} 1257)$ indicated the sialic acid was $\alpha 2,3$-linked to the galactose prior to digestion. The core GalNAc was sialylated via an $\alpha 2,6$-linkage which could not be removed by neuraminidase $S$ and therefore generated the mono-sialylated core structure $(\mathrm{m} / \mathrm{z} 896)$ after the enzymatic digest. The residual peak that represented a di-sialylated structure at $\mathrm{m} / \mathrm{z} 1257$ confirmed the presence of a di-sialyl motif that was resistant to neuraminidase $S$ treatment (Fig. $4 \mathrm{c}, \mathrm{m} / \mathrm{z} 1256$ ).

Glycosphingolipid-derived glycome expresses sialylated structures with linear elongation. Glycosphingolipids (GSLs) represent a major class of glycans that are present on the plasma membrane. 
a No treatment

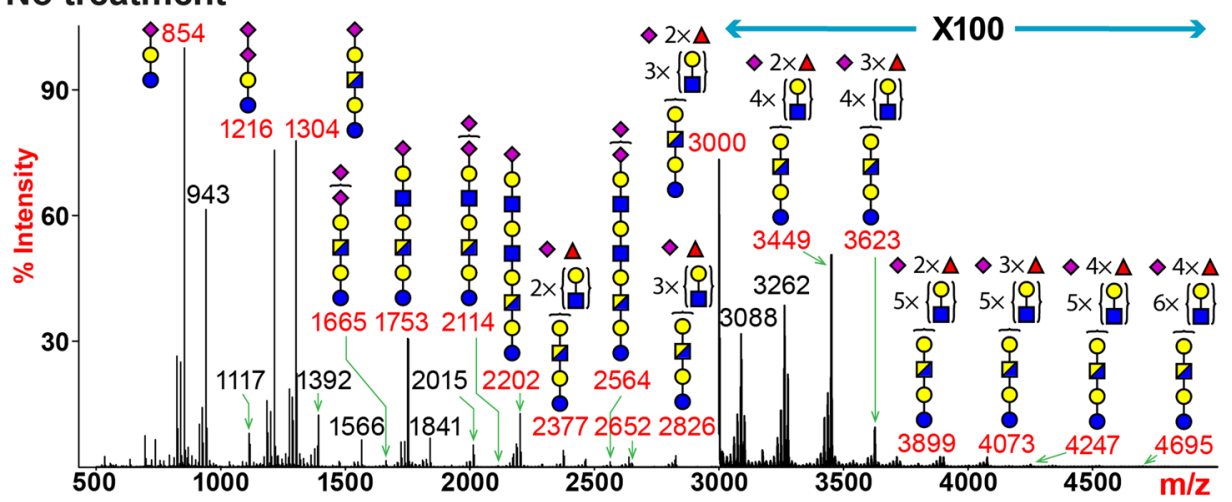

b Treatment with neuraminidase A ( $\alpha 2,3 / 6 / 8 / 9$-linkage specific)

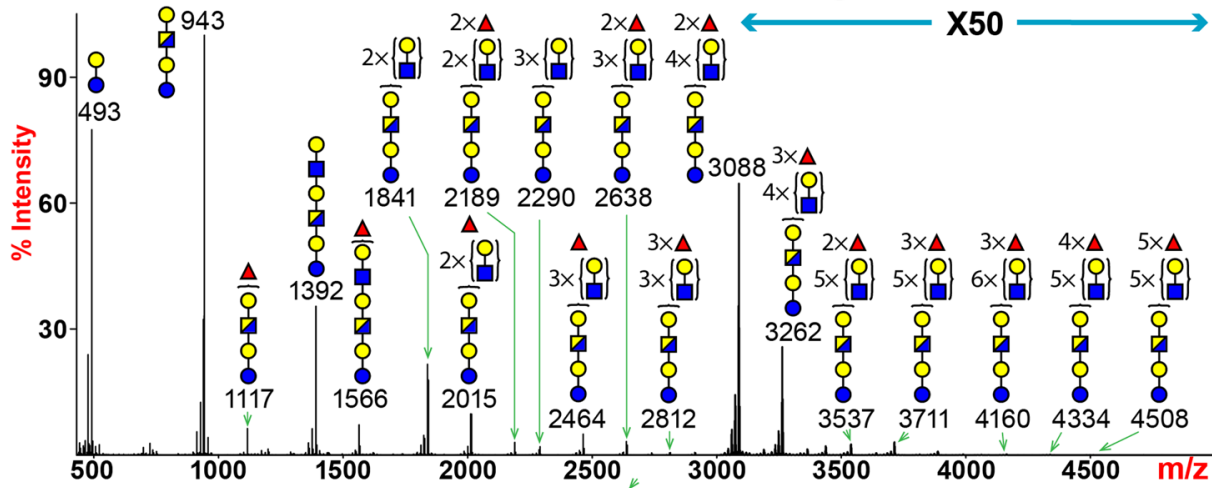

c Treatment with neuraminidase $S$ ( $\alpha 2,3$-linkage specific)

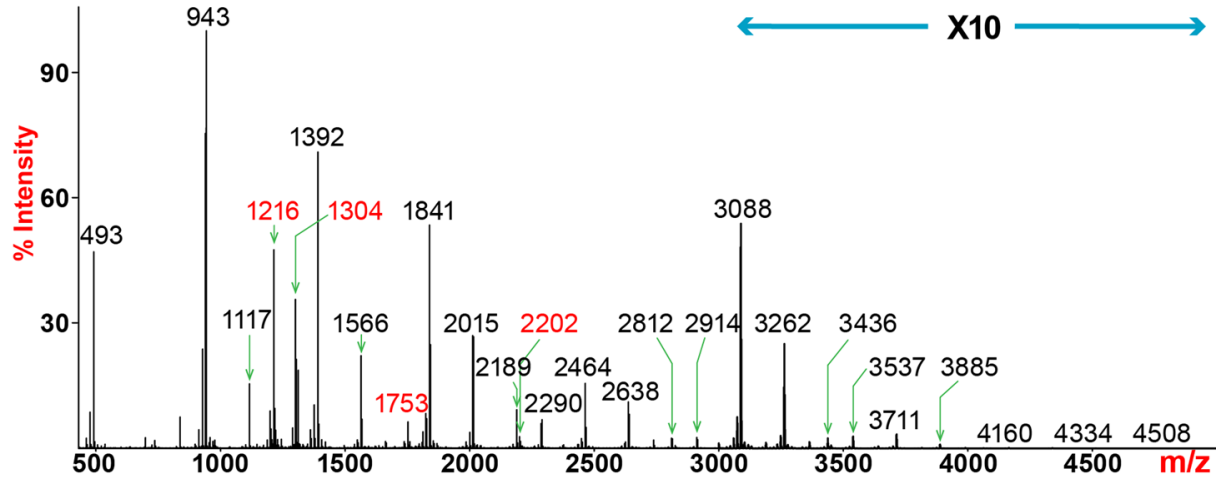

Figure 5. Glycosphingolipid (GSL)-glycan MS profiles of human lung following neuraminidase treatment. All molecular ions detected represent reduced, permethylated species and are present in the form of $[\mathrm{M}+\mathrm{Na}]^{+}$. (a) MALDI-TOF-MS spectrum of untreated human lung GSL-glycans. (b) MALDI-TOF-MS spectrum of human lung GSL-glycans treated with neuraminidase S. (c) MALDI-TOF-MS spectrum of human lung GSL-glycans treated with neuraminidase A. Peaks representing sialylated glycans are colored in red and non-sialylated glycans are in black. Symbol representations of monosaccharides are: yellow circle, Galactose; red triangle, Fucose; blue square, $\mathrm{N}$-acetylglucosamine; half-blue/half-yellow square, $\mathrm{N}$-acetylhexosamine; purple diamond, $\mathrm{N}$-acetylneuraminic acid.

Through MALDI-TOF MS analysis of human lung GSL-glycome, we detected more than 40 unique assignable peaks (Fig. 5a; Supplementary Table 4). Molecular ions consistent with the compositions of GM3 (ganglioside series, mono-sialylated glycan-3, m/z 854), GD3 (ganglioside series, di-sialylated glycan-3, m/z 1216) and GM1 (ganglioside series, mono-sialylated glycan-1, $\mathrm{m} / \mathrm{z}$ 1304) represented the most abundant structures. The peak at $\mathrm{m} / \mathrm{z} 943$ corresponded to a structure with the composition of Gal-HexNAc-Gal-Glc, which represented the most abundant non-sialylated glycan (Fig. 5a). Sequential extensions of this tetra-saccharide core with variable lengths of PL repeats generated a series of unmodified (m/z 1392 and 1841), fucosylated (m/z 1117, 1566, 2015, 3088 and 3262) or sialylated (m/z 1753, 2202 and 2564) glycans. Towards the higher $\mathrm{m} / \mathrm{z}$ region, molecular ions corresponding to sialylated structures with the addition of one or more fucoses were detected $(\mathrm{m} / \mathrm{z}$ 2377, 2826, 3000, 3449, 3623, 3899, 4073, 4247 and 4695). MS/MS analysis of the molecular ion at $\mathrm{m} / \mathrm{z} 3449$ revealed that the LacNAc extensions beyond the Gal-HexNAc-Gal-Glc core occurred in a linear fashion, rather 
a $\mathrm{N}$-glycan

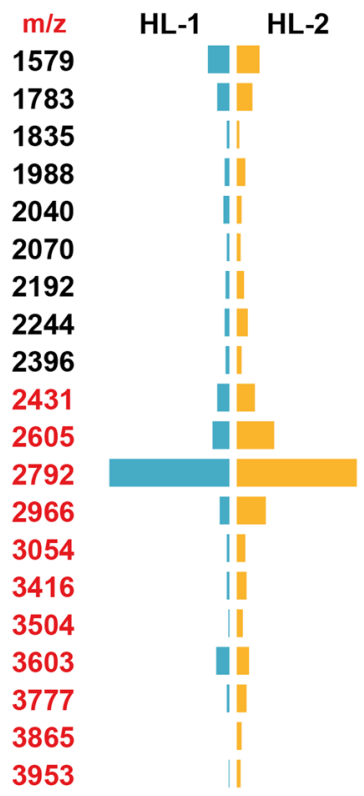

Human lung-1

Human lung-2 b O-glycan

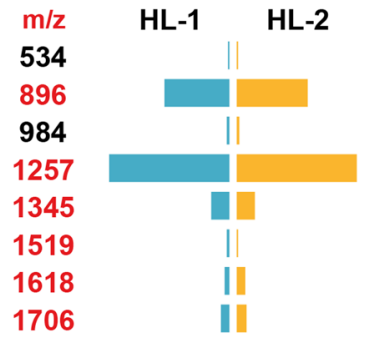

c GSL-glycan

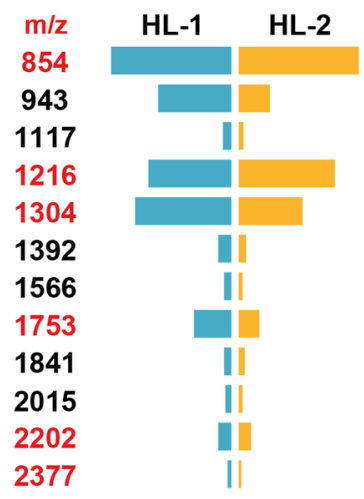

Figure 6. Comparison of glycosylation patterns between two human lungs. The relative intensities of selected N-glycans (a), O-glycans (b) and GSL-glycans (c) expressed by two human lungs are presented in a diverging bar chat. The length of a bar is proportional to the relative intensity $(0-100 \%)$ of a peak detected on a MALDITOF-MS spectrum. A blue bar indicates the glycan is expressed by human lung-1 and an orange bar indicates the glycan is expressed by human lung-2. Peaks representing sialylated glycans are colored in red and nonsialylated glycans are in black.

than branched (Supplementary Fig. 6a,b). Sialic acids only appeared on the non-reducing terminus in the form of Neu5Ac-LacNAc or SLeA/X antigen, with the latter isomeric structure being a minor population (Supplementary Fig. 6a). Additional fucose residues were linked to the repeating LacNAc units, generating an internal LeX motif, similar to what had been observed for human lung $\mathrm{N}$-glycans. The common structural features of linear extension, terminal sialylation and the expression of internal LeX antigens were also shared by other glycans (e.g., $\mathrm{m} / \mathrm{z}$ 2377, 2826 and 3000) and confirmed with MS/MS experiments.

Neuraminidase A digest completely removed sialic acids, revealing the pattern of LacNAc extension and fucosylation (Fig. 5b). The MALDI-TOF/TOF MS/MS spectrum of the molecular ion at $\mathrm{m} / \mathrm{z} 3262$ showed linear elongation of LacNAc units, as we observed fragment ions representing sequential addition of LacNAc or Fuc-LacNAc to the Gal-Glc core from the reducing end or to the terminal LeA/X antigen from the non-reducing end (Supplementary Fig. 6b). Neuraminidase S treatment fully desialylated GM3 (Fig. 5c, m/z 854), confirming the sialylation was in $\alpha 2,3$-linkage. In contrast, the outermost sialic acid of GD3 (m/z 1216) was in $\alpha 2,8$-linkage and therefore was resistant to hydrolysis. Partial desialylation of mono-sialylated glycans (m/z 1304, 1753 and 2202 ) indicated the expression of both $\alpha 2,3$ - and $\alpha 2,6$-linkages. Glycans detected in the higher $\mathrm{m} / \mathrm{z}$ region with four or more LacNAc units were completely desialylated, indicating those structures with PL extensions were entirely $\alpha 2,3$-sialylated prior to neuraminidase $S$ digest $(\mathrm{m} / \mathrm{z} 2377,2564,2826,3000,3449,3623,3899,4073$, 4247 and 4695). The pattern of differential sialylation recapitulated what had been observed for N-glycans where $\alpha 2,6$-sialylation preferentially occurs on smaller-sized glycans and larger-sized glycans with PL extensions are predominantly $\alpha 2,3$-sialylated.

Characterization of a second human lung revealed similar patterns of glycosylation. To assess whether the glycosylation patterns we observed from one person represented common structural features in other individuals, we characterized the glycome of a second human lung. The Coomassie blue staining of the second lung showed similar patterns of protein expression to that of the first lung (Supplementary Fig. 7a). The expressions of sialylated and fucosylated structures were confirmed by staining with lectins and antibodies of homogenates from the second lung (Supplementary Fig. 7b-j). MS analysis revealed that the overall patterns of $\mathrm{N}$-glycosylation were highly comparable between the two lungs with the bi-antennary, di-sialylated structure $(\mathrm{m} / \mathrm{z}$ 2792) remained the most abundant glycan in the second lung (Fig. 6a, Supplementary Fig. 8a). Molecular ions consistent with a series of sialylated, fucosylated and bisected species were detected up to $\mathrm{m} / \mathrm{z} 12000$. As expected, some marginal differences were observed, which were likely due to person-to-person variations in the expression levels of glycosyltransferases (e.g., $\mathrm{m} / \mathrm{z}$ 2605, 2966, 3054, 3416 and 3777). We did not detect unique 
a

SNA

MAL-I

$\mathrm{X}-31$

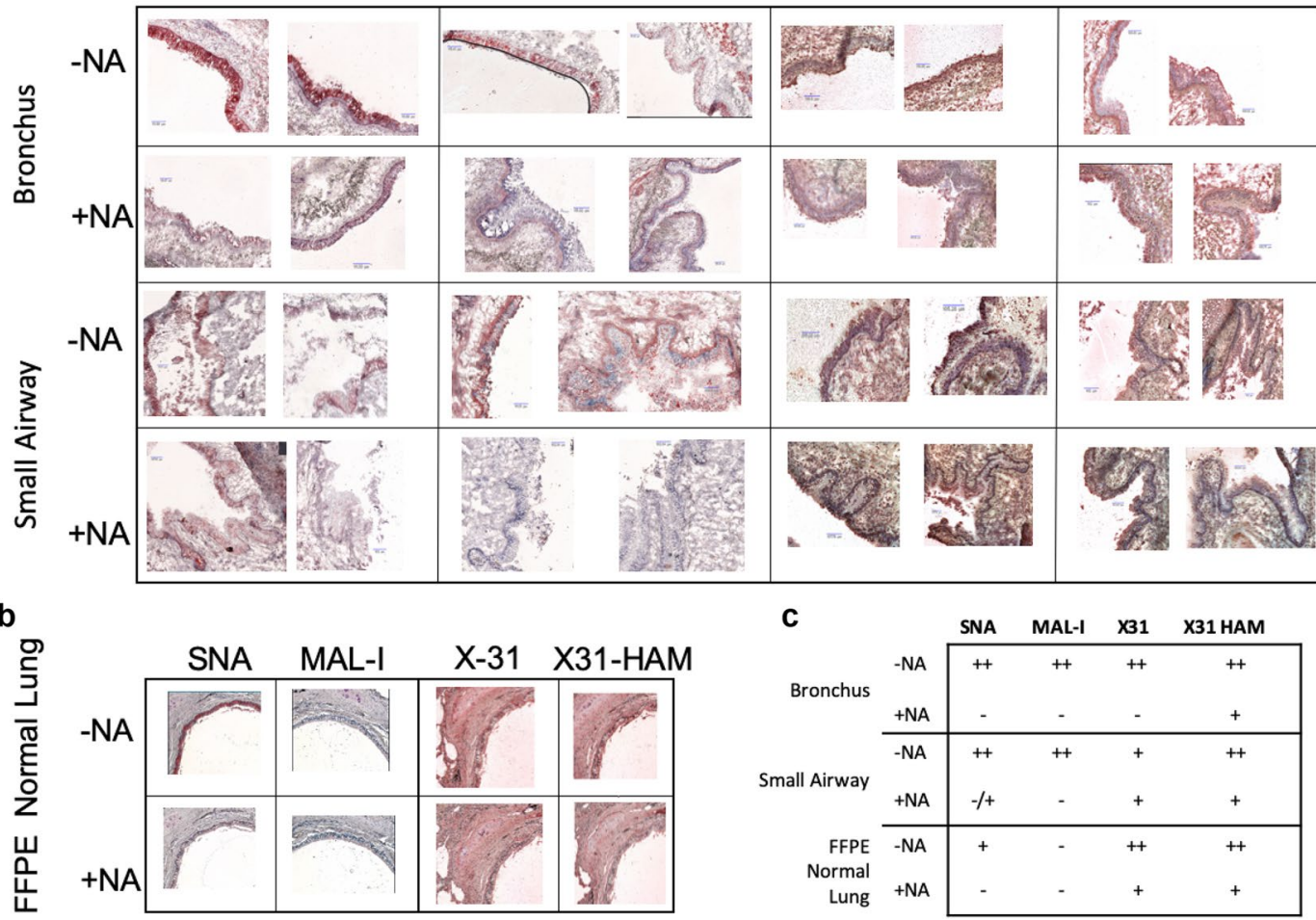

Figure 7. Immunohistochemistry staining of human lung sections. To visualize the localization of $\alpha 2,3-$ and $\alpha 2,6$-linked sialic acids and the patterns of influenza virus attachment, human respiratory tissue sections were stained with lectins and influenza viruses. (a) Lectin-histochemistry staining images of frozen human bronchus and small airway sections with lectins SNA and MAL-I or with X-31 and X31-HAM strains of influenza viruses before ( $-\mathrm{NA})$ or after ( $+\mathrm{NA})$ neuraminidase treatment. (b) Lectin-histochemistry staining images of formalin fixed paraffin embedded (FFPE) human lung sections with lectins SNA and MAL-I or with X-31 and X31HAM strains of influenza viruses before ( $-\mathrm{NA}$ ) or after (+NA) neuraminidase treatment. (c) Summary of immunohistochemistry staining results. ++ , strong staining; + , moderate staining; $-/+$, weak staining; - , no staining.

composition that only belonged to one lung but not the other. The O-glycosylation patterns were almost identical between the two lungs (Fig. 6b, Supplementary Fig. 8b) and the profiles for GSL-glycans were also comparable (Fig. 6c, Supplementary Fig. 8c). Mono- and di-sialylated structures represented the major species (e.g., $\mathrm{m} / \mathrm{z} 854$, $1216,1304,1753$ and 2202) while multi-fucosylation occurred on structures with variable PL repeats (e.g., $\mathrm{m} / \mathrm{z}$ $3000,3088,3262,3449,3623$ and 4073). The level of a non-sialylated glycan (m/z 943) was notably lower in the second lung. Overall, these results indicate that the glycosylation pattern of human lungs is likely to be similar between individuals, yet some degree of person-to-person variation could occur.

Human lung tissue sections revealed staining with lectins and influenza viruses. The glycomics analysis examines the total lung glycome without specific localization to tissue types. Human lung tissue slides of the bronchus and small airways, from the donor organs prior to homogenization for glycomics analysis, were prepared for lectin histochemistry and pattern of virus attachment studies to aid in the characterization of sialylated receptors localized to these areas of the lung. On the frozen tissue slides (Fig. 7a), epithelial cells stained strongly without distinct localization for SNA for both tissue types, whereas MAL-I stained strongly on the basal surface with spotty staining at the apical edge in small airways. Bronchus epithelial cell staining is more uniform. Neuraminidase A (NA) treatment revealed that potential MAL-I binding to non-sialylated N-glycans does not provide a significant contribution to the overall lectin staining. Commercially available formalin fixed, paraffin embedded (FFPE) slides of normal human lung were used for comparison studies, and only SNA binding was detectable and was removed following NA treatment. MAL-I binding was not detected. Such discrepancies in results for frozen versus FFPE slides have previously been reported and could be due to the differences in slide preparation and fixation ${ }^{23,33-39}$ (Supplementary Table 5). It is possible that the frozen slides retain glycolipids, with a predominance of $\alpha 2,3$-linked Neu5Ac, that are removed in the fixation process for FFPE slides. It should also be noted that the Maackia amurensis preparation used in the various studies referenced here is not consistent; some studies use one variation of the lectin (MAL-I or MAL-II) and others utilize a combination (MAA).

Virus attachment experiments were performed to examine the surface attachment of IAV to the cells of the frozen and fixed lung tissue sections ${ }^{40}$ (Fig. 7b,c). We used X-31 and X-31 HAM viruses for these assays, as they exhibit strict sialic acid linkage preference for binding. X-31 recognizes $\alpha 2,6-$ sialic acid and X-31 HAM 
recognizes $\alpha 2,3$ - sialic acid ${ }^{41}$, thereby providing localization information in addition to broad confirmation of the presence of sialylated receptors. Both viruses also bind to all frozen and fixed tissue sections. Neuraminidase treatment effectively prevents staining by lectins to the frozen tissue slides, however some viral attachment is detectable following the same neuraminidase treatment conditions, revealing the possibility that the viruses are recognizing non-sialylated receptors in addition to the canonical sialylated ones which were removed by neuraminidase treatment ${ }^{24}$.

Antibodies against LeA, LeX, SLeA and SLeX were used in immunofluorescence experiments with frozen small airway sections (Supplementary Fig. 9). The anti-LeA antibody showed no staining to the epithelium of the small airway, while punctate staining was observed for anti-LeX and anti-SLeA antibodies. The antibody against SLeX exhibited strong staining to the epithelial cells corroborating MS data with the detection of SLeX epitope. Interestingly, while the LeA antibody recognized distinct glycoproteins on the Western blots, we did not detect staining to the epithelial cells via immunofluorescence, suggesting that such O-glycans are not predominant on such cells.

\section{Discussion}

Here we present a comprehensive analysis of the natural N-, O- and GSL-glycans expressed by a human lung. Our MS data suggested that there appears to be a differential expression of $\alpha 2,3$ - versus $\alpha 2,6$-sialylated glycans. N- and GSL-glycans found in a human lung are linearly extended with variable lengths of PL repeats with $\alpha 2,6$-sialylation predominantly occurring on short PL sequences and $\alpha 2,3$-sialylation on long PL sequences, perhaps revealing substrate preference for the $\alpha 2,3$ - and $\alpha 2,6$-sialyltransferases. According to previous studies, the kinetic $\mathrm{K}_{\mathrm{m}}$ value of the human $\alpha 2,6$-sialyltransferase-I (ST6Gal-I) towards a single type 2 LacNAc unit is significantly lower than that of the human $\alpha 2,3$-sialyltransferase-III, IV and VI (ST3Gal-III, IV and VI), which suggests that the ST6Gal-I may have a higher affinity towards short LacNAc sequences ${ }^{42,43}$. The crystal structure of ST6Gal-I further supports this speculation, as the active site of the enzyme has limited space to accommodate extended glycan sequences and the tri-mannosyl core of $\mathrm{N}$-glycans appears to be critical for substrate recognition $^{44}$. However, differential sialylation can also be affected by the expression levels of the sialyltransferases and their relative localizations within a cell.

The structural information of those natural glycans is crucial to understand the initial infection process by pulmonary pathogens that use glycans as host receptors. IAV strains can bind to sialic acid-terminating glycans expressed on the surface of human airway and the linkage difference of sialic acids is thought to be a key factor for the species barrier of influenza infection. This simple paradigm has evolved in recent years to include the glycan sequences beyond the terminal sialic acids. Sialylated branched N-glycans with elongated PL units have been proposed to be involved in IAV recognition ${ }^{45-47}$. Even though many studies have been using immuno-lectin histochemistry methods, glycan microarray platforms or computational tools to interrogate the molecular determinants governing the glycan-virus recognition, little structural information has been known for glycans naturally present in human respiratory tissues ${ }^{21,23,45,48,49}$. Therefore, our current understanding may not fully reflect the biological context of influenza infection on the surface of human airway. Our work confirmed the presence of $\alpha 2,6$-sialylated $\mathrm{N}$-glycans with short LacNAc repeats $(\mathrm{n} \leq 3)$ in human lungs. Those structures have been used in many glycan microarray studies and have shown binding to various strains of influenza viruses ${ }^{45,50-53}$. However, even though $\alpha 2$,6-sialylated N-glycans with extended PL $(n>3)$ sequences exhibit binding to H3N2 strains of influenza viruses ${ }^{45}$, there is weak evidence to support their expression by human, ferret or swine respiratory tissues $^{21,54,55}$. On the contrary, $\alpha 2,3$-sialylated $\mathrm{N}$-glycans with long PL $(\mathrm{n} \geq 4)$ repeats, which were identified from the present work, are poor binders for influenza viruses ${ }^{45,53}$. Staining of human lung tissue slides indicates that neuraminidase treatment removing all sialic acid detectable by lectin binding does not prevent attachment of the influenza viruses X-31 and X-31 HAM. Moreover, our recent study identified a novel mode of glycan recognition by influenza viruses, which depends on phosphorylated glycans ${ }^{24}$. These results open up possibilities that influenza viruses may rely on multiple types of host receptors to adhere to respiratory epithelial cells and the infection process may be more complicated than currently supposed.

The tri-sialylated core $1 \mathrm{O}$-glycan has been associated with cytotoxicity of natural killer (NK) cells via inhibitory cis-interaction with Siglec-7 on cell surface ${ }^{56,57}$. The enzyme responsible for the addition of an $\alpha 2,8$-linked Neu5Ac on O-glycans has been shown to be human $\alpha 2,8$-sialyltransferase-VI and its expression level is enhanced in a human lung (www.proteinatlas.org). We speculate that a human lung may be armed with a huge number of tissue-resident NK cells to protect the lung from constant encounter of respiratory pathogen ${ }^{58,59}$. On the other hand, the enhanced expression of the tri-sialylated O-glycan may contribute to the hypo-responsiveness of NK cells so that they are not too damaging to lung tissues.

Our comprehensive characterization of human lung glycome also allows us to compare and verify animal models used in the study of influenza infection, from the angle of pulmonary glycosylation. By recruiting the same strategy of glycan release and MS analysis, the glycomic profiles of lungs from ferret, mouse and swine have been generated from previous studies using total lung organs as starting materials ${ }^{54,55,60}$. The ferret glycome has many structural features in common with the glycome of human lung, exhibiting a comparable MS profile, presence of moderate lengths of PL, exclusive expression of the Neu5Ac form ${ }^{61}$ and sialylation with a higher proportion towards $\alpha 2,6$ - than $\alpha 2,3$-linked Neu5Ac. However, the expression of the Sda antigen (Neu5Aco2,3(GalNAc $\beta 1-4)$ $\mathrm{Gal} \beta 1,4 \mathrm{GlcNAc}$ ) represents a major and unique structural feature of ferret lung glycome. The analysis of mouse lung revealed a highly comparable $\mathrm{N}$-glycan profile to that of human lung, except the sialylation is predominantly in the form of Neu5 $\mathrm{Gc}^{60}$. Despite the high degree of similarity, the mouse has been regarded as a poor animal model for the studies of influenza viruses, possibly due to poor binding affinity by influenza viruses against the $\mathrm{Neu} 5 \mathrm{Gc}^{62}$. On the other hand, the glycome of a swine lung exhibited less similarity to a human lung, as the swine lung expresses high abundances of structures carrying the Neu5Gc and Gala1-3Gal non-human epitopes. 
However, swine are susceptible to infection by human, avian and swine influenza viruses. Additional factors such as the composition of pulmonary mucus may contribute to the difference in infectivity. Although not in the scope of the present study, human airway mucins are known to contain hundreds of sialylated, fucosylated and sulfated glycan structures ${ }^{32}$. In comparison to the data obtained from the present study, the O-glycome of a human lung is relatively simple.

In summary, the glycomics analysis of the human lung has generated a wealth of data with implications in influenza research, immunological studies, and technical advances including the analysis of phosphorylated glycans via MALDI-TOF MS. This work represents a beginning for the Human Glycome Project and paves the way for the study of other tissues and organs in the future.

\section{Materials and Methods}

Materials. Human donor lungs unfit for organ transplantation were provided by LifeLink Foundation, Inc. (Norcross, GA). The lungs were obtained through LifeLink, one of the approved non-profit organ procurements organizations, through informed and consented organ donation of deceased individuals and are not considered human subjects research. No personal identifying information was obtained on the donors, and falls under exemption category \#4 (2016P000014), through the Beth Israel Deaconess Medical Center IRB. The lung\#1 used in the present study for extensive glycomics analysis was obtained from a healthy 22 -year old male without any pulmonary complications. The lung\#2 was obtained from a healthy 19 -year old male with minimal smoking history. Human lungs used for analyses were perfused before receipt and devoid of blood cells and soluble mucus. Materials were treated following ethical guidelines and regulations and approval for work with human tissues was obtained through Beth Israel Deaconess Medical Center and Harvard Medical School (COMS approval \#15-251).

Western blots. The human lung homogenate was prepared by blending the tissues in lysis buffer $(25 \mathrm{mM}$ TRIS, $150 \mathrm{mM} \mathrm{NaCl}, 5 \mathrm{mM}$ EDTA, 1\% CHAPS, pH 7.4), followed by sonication. Digestion of the homogenate by PNGase F, neuraminidase A and neuraminidase $S$ was performed at $37^{\circ} \mathrm{C}$ for 16 hours (New England Biolabs, Inc.). Proteins were stained with Coomassie Brilliant Blue (CBB) or transferred to a nitrocellulose membrane (Thermo Fisher Scientific). After blocking with $5 \%$ (w/v) bovine serum albumin (BSA) in TRIS-buffer saline with 0.05\% TWEEN 20 (TBST) for $1 \mathrm{~h}$ at room temperature, Western and lectin blots were analyzed with anti-LeA (7LE, Santa Cruz Biotechnology), anti-SLeA (9L426, US Biological), anti-LeX (HI98, BioLegend, Inc.), biotinylated AAL, ConA, DBA, MAL-I, SNA, or UEA-I (Vector Laboratories) as a primary staining, and horseradish peroxidase (HRP)-labelled goat anti-mouse IgG antibody, goat anti-mouse IgM antibody, or streptavidin at 1:5000 dilution in TBST, using SuperSignal West Pico Chemiluminescent Substrate (Thermo Fisher Scientific).

General procedures for glycan release. Detailed sample preparation procedures for glycomics analysis by mass spectrometry have been described previously ${ }^{63}$. In brief, frozen human lung tissue was homogenized and sonicated in ice-cold ultra-pure water. Glycolipids were extracted by the addition of methanol and chloroform whereas glycoprotein precipitants were collected by centrifugation. Glycans were released from GSLs via a recombinant endoglycoceramidase II (rEGCase II; Takara Bio USA Inc.) and reduced with sodium borohydride. Glycopeptides were reduced and carboxymethylated with dithiothreitol and iodoacetamide, followed by overnight trypsin digestion. N-linked glycans were released from glycopeptides by PNGase F and O-linked glycans by reductive elimination. All glycans were permethylated, chloroform extracted and purified by C18 Sep-Pak pre-packed columns (WAT054945, Waters Corp.) prior to mass spectrometric analysis.

Neuraminidase digestion. To acquire the linkage information of sialylation, purified glycans were incubated with neuraminidase A (from Arthrobacter ureafaciens, P0722, New England Biolabs, Inc.) or neuraminidase S (from Streptococcus pneumoniae, P0743, New England Biolabs, Inc.) over a period of 24 hours at $37^{\circ} \mathrm{C}$.

Sample preparation for phosphoglycomics. Following tryptic digestion of human lung homogenates, glycopeptides were treated with Endo H (from Streptomyces picatus, P0702, New England Biolabs, Inc.) over a period of 24 hours at $37^{\circ} \mathrm{C}$. Released glycans were separated from residual glycopeptides via C18 chromatography and were subsequently desialylated by mild acid hydrolysis $\left(50 \mathrm{mM}\right.$ TFA, $20 \mathrm{~min}$ at $\left.100^{\circ} \mathrm{C}\right)$. Hydrolyzed glycans were fractionated into non-charged and charged pools via a strong-anion exchanger (QAE Sephadex A-25, GE Life Sciences). Neutral glycans were present in the flow-through and wash fractions (20 mM TRIS-base). Bound phosphorylated glycans were eluted with $100 \mathrm{mM} \mathrm{NaCl}$ in $20 \mathrm{mM}$ TRIS buffer. Once dried, both charged and non-charged fractions can be permethylated directly.

Data acquisition by mass spectrometer. The permethylated glycans were dissolved in $20 \mu \mathrm{l}$ of methanol, from which $1 \mu \mathrm{l}$ of the sample was mixed with $1 \mu \mathrm{l}$ of the matrix $(20 \mathrm{mg} / \mathrm{ml}$ of 2,5 -dihydrobenzoic acid in $50 \%(\mathrm{v} / \mathrm{v})$ aqueous methanol) and spotted onto a MTP 384 polished steel BC target plate (Bruker Daltonics). MALDI-TOF MS and MALDI-TOF/TOF MS/MS data were obtained from an ultrafleXreme mass spectrometer (Bruker Corp.) equipped with a Smartbeam II laser. Data acquisition was performed under positive mode via the software flexControl (version 3.4, build 135, Bruker Daltonics). Spectrum between mass-to-charge ( $\mathrm{m} / \mathrm{z}$ ) 1000 and 8000 was acquired under reflectron mode whereas between m/z 5000 and 12000 was acquired under the linear mode. Monoisotopic masses are listed for compositions with $\mathrm{m} / \mathrm{z}$ between 1000 and 7000 while average masses are listed for $\mathrm{m} / \mathrm{z}$ between 5000 and 12000. The ProteoMass MALDI-MS calibration kit (MSCAL1, Sigma-Aldrich) was used to calibrate the MS mode. Each MS spectrum presented in this study represented an accumulated spectrum that was generated from 20,000 laser shots. 
Data processing and peak assignment. MS and MS/MS raw data was exported from flexAnalysis (version 3.4, build 76, Bruker Daltonics) as mass spectrum and was further processed by mMass ${ }^{64}$. Each mass spectrum was assigned and annotated manually with the aid of GlycoWorkBench ${ }^{65}$. The assignment of a glycan composition below $\mathrm{m} / \mathrm{z} 7000$ was based on the ${ }^{12} \mathrm{C}$ isotopic composition of a selected peak as well as the knowledge of biosynthetic pathways of mammalian glycans. For peaks above $\mathrm{m} / \mathrm{z} 7000$, average mass was used to annotate the composition of a peak. Peak assignment was reassured by the detection of mass shift that corresponded to the composition of a LacNAc, a GlcNAc, a Fuc or a Neu5Ac. All N-glycans observed in this study were assumed to have a common core sequence of Man $\alpha 1-6(\operatorname{Man} \alpha 1-3)$ Man $\beta 1-4 G l c N A c \beta 1-4 G l c N A c$. All O-linked glycans were assumed to be mucin-type glycans with a reducing end GalNAc common core attached to Thr or Ser. All glycolipid-derived glycans were assumed to be the glycosphingolipid type glycans with a common core sequence of Gal $31-4 \mathrm{Glc}$. Wherever possible, a proposed structure was further sequenced by MS/MS experiments to define additional structural information.

Percentage calculation of structural features. The relative intensity of the most abundant peak in each spectrum was set at $100 \%$ and all other peaks were normalized accordingly. To generate the doughnut chart that illustrates the total percentage of a specific structural feature, the following equation was used:

$$
\text { Total } \% \text { of a structural feature }=\frac{\sum \text { relative } \% \text { of all molecular species that contain the structural feature }}{\sum \text { relative } \% \text { of all molecular species }}
$$

The $\mathrm{m} / \mathrm{z}$ values of molecular species used in the calculation range between 1000 and 7000 .

GC-MS linkage analysis. Detailed procedures have been previously described ${ }^{63}$. In brief, permethylated glycans were hydrolyzed in TFA before reduction by sodium borodeuteride and acetylation with acetic anhydride. The resulting partially permethylated alditol acetates were dissolved in hexane and analyzed by a Thermo Scientific TRACE 1310 Gas Chromatograph equipped with a Thermo Scientific Q Exactive Orbitrap mass spectrometry system. $2-3 \mu \mathrm{L}$ of each sample was injected into an Agilent fused-silica capillary column of cross-linked DB-5MS $(30 \mathrm{~m} \times 0.25 \mathrm{~mm} \times 0.25 \mu \mathrm{m})$. The GC conditions were as follows: inlet and transfer line temperatures, $290^{\circ} \mathrm{C}$; oven temperature program, $90^{\circ} \mathrm{C}$ for $1 \mathrm{~min}, 8^{\circ} \mathrm{C} / \mathrm{min}$ to $290^{\circ} \mathrm{C}$ for $5 \mathrm{~min}, 10^{\circ} \mathrm{C} / \mathrm{min}$ to $300^{\circ} \mathrm{C}$ for $5 \mathrm{~min}$; inlet helium carrier gas flow rate, $1 \mathrm{~mL} / \mathrm{min}$; split ratio, 10 . The electron impact (EI)-MS conditions were as follows: ion source temperature, $300^{\circ} \mathrm{C}$; full scan $\mathrm{m} / \mathrm{z}$ range, $30-750 \mathrm{Da}$; resolution, 60,000; AGC target, 1e6; maximum IT, $200 \mathrm{~ms}$. Data were acquired and analyzed with Thermo TraceFinder 4.1 software package.

Immuno-histochemistry staining. Frozen sections of human lung tissue were fixed in acetone: ethanol $(75: 25)$ prior to staining. Slides are pre-treated with hydrogen peroxide and blocked with $5 \%$ BSA in Phosphate-Buffered Saline with Tween-20 (PBST, 0.05\% Tween). After 45 min washes in PBST, slides were incubated with biotinylated SNA or MAL-I (both at $20 \mathrm{ug} / \mathrm{ml}$ ) overnight at $4{ }^{\circ} \mathrm{C}$. The lectins were removed and the slides washed in PBST prior to incubation in Streptavidin-HRP $(1: 1000)$ for 1 hour at room temperature. A Tyramide Signal Amplification (TSA) kit was used to amplify the HRP signal ${ }^{34}$. Patterns of IAVs attachment were conducted in a similar manner with the blocking step using 5\% normal goat serum in PBST and FITC-labelled virus $^{40}$ as the primary. Rabbit anti-FITC HRP was used for secondary detection, TSA TMR reagent was applied for $10 \mathrm{~min}$ and the signal was developed for $2 \mathrm{~min}$ in AEC. Cells were counter stained with hematoxylin. Formalin fixed paraffin embedded slides (US Biomax, Inc.) were deparaffinized with xylene and rehydrated with an ethanol gradient. Antigen retrieval was completed by microwaving the slides in citrate buffer $(0.1 \mathrm{M}$ citrate acid, $0.1 \mathrm{M}$ sodium citrate, $\mathrm{pH}$ 6.0) for $15 \mathrm{~min}$. Slides were then stained in the same way as the frozen section slides.

Immunofluorescence staining with antibodies. The double immunofluorescence antigen labelling of frozen human lung sections was performed with LeA, LeX, SLeA and SLeX, with EpCAM antibody. The frozen human lung sections were fixed in Acetone/Ethanol (75:25) for $10 \mathrm{~min}$ and washed twice with TBS. The sections were blocked with 5\% normal donkey serum (Jackson ImmunoResearch Lab Inc, West Grove PA) for an hour at room temperature. The sections were incubated with rabbit anti-EpCAM (1:300, Abcam, Cat\#: ab32392) plus (1) FITC conjugated mouse anti-LeA (1:100, Santa Cruz, cat\#: sc-51512) and (2) FITC conjugated mouse anti-human CD15 (LeX, 1:50); (3) mouse anti-SLeA (1:500, CA19.9) and (4) Rat anti-SLeX (1:200, BD 555946) overnight at $4{ }^{\circ} \mathrm{C}$. The slides were washed three times in TBS and incubated with Alexa 647 conjugated donkey anti-rabbit secondary antibodies (Jackson ImmunoResearch Lab, 1:300) for (1) and (2) and CY3 conjugated donkey anti-mouse (Jackson ImmunoResearch Lab, 1:300) and Alexa 647 conjugated donkey anti-rabbit secondary antibodies (Jackson ImmunoResearch Lab, 1:300) for (3) and CY3 conjugated donkey anti-rat (Jackson ImmunoResearch Lab, 1:300) and Alexa 647 conjugated donkey anti-rabbit secondary antibodies (Jackson ImmunoResearch Lab, 1:300) for (4). Samples were then washed three times with TBS and mounted with Prolong Gold anti-fade mounting media containing DAPI (Invitrogen).

Reporting summary. Further information on research design is available in the Nature Research Reporting Summary linked to this article.

\section{Data availability}

All data generated or analyzed during this study are included and in this published article and its Supplemental Information files.

Received: 6 December 2019; Accepted: 28 February 2020;

Published online: 24 March 2020 


\section{References}

1. Apweiler, R., Hermjakob, H. \& Sharon, N. On the frequency of protein glycosylation, as deduced from analysis of the SWISS-PROT database. Biochim. Biophys. Acta 1473, 4-8 (1999).

2. Varki, A. Essentials of glycobiology. Third edition/Edited by Ajit Varki et al. Cold Spring Harbor Press (2017).

3. Xu, C. \& Ng, D. T. Glycosylation-directed quality control of protein folding. Nat. Rev. Mol. Cell Biol. 16, 742-752 (2015).

4. Hardivillé, S. \& Hart, G. W. Nutrient regulation of signaling, transcription, and cell physiology by O-GlcNAcylation. Cell Metab. 20, 208-213 (2014).

5. Macauley, M. S., Crocker, P. R. \& Paulson, J. C. Siglec-mediated regulation of immune cell function in disease. Nat. Rev. Immunol. 14, 653-666 (2014).

6. Cummings, R. D. The repertoire of glycan determinants in the human glycome. Mol. Biosyst. 5, 1087-1104 (2009).

7. Schachter, H. \& Freeze, H. H. Glycosylation diseases: quo vadis? Biochim Biophys Acta 1792, 925-930 (2009).

8. Haslam, S. M. et al. Characterizing the glycome of the mammalian immune system. Immunol. Cell Biol. 86, 564-573 (2008).

9. Seeling, M., Brückner, C. \& Nimmerjahn, F. Differential antibody glycosylation in autoimmunity: sweet biomarker or modulator of disease activity? Nat. Rev. Rheumatol. 13, 621-630 (2017).

10. Kunz, C., Rudloff, S., Baier, W., Klein, N. \& Strobel, S. Oligosaccharides in human milk: structural, functional, and metabolic aspects. Annu. Rev. Nutr. 20, 699-722 (2000).

11. Sharapov, S. Z. et al. Defining the genetic control of human blood plasma N-glycome using genome-wide association study. Hum. Mol. Genet. 28, 2062-2077 (2019).

12. Möginger, U. et al. Alterations of the Human Skin. Front. Oncol. 8, 70 (2018).

13. Gizaw, S. T., Ohashi, T., Tanaka, M., Hinou, H. \& Nishimura, S. Glycoblotting method allows for rapid and efficient glycome profiling of human Alzheimer's disease brain, serum and cerebrospinal fluid towards potential biomarker discovery. Biochim. Biophys. Acta 1860, 1716-1727 (2016).

14. Sethi, M. K. et al. In-depth N-glycome profiling of paired colorectal cancer and non-tumorigenic tissues reveals cancer-, stage- and EGFR-specific protein N-glycosylation. Glycobiol. 25, 1064-1078 (2015).

15. Li, Q. et al. Comprehensive N-Glycome Profiling of Cells and Tissues for Breast Cancer Diagnosis. J. Proteome Res. 18, 2559-2570 (2019).

16. Thompson, A. J., de Vries, R. P. \& Paulson, J. C. Virus recognition of glycan receptors. Curr. Opin. Virol. 34, 117-129 (2019).

17. Robb, M. et al. Molecular Characterization of N-glycan Degradation and Transport in Streptococcus pneumoniae and Its Contribution to Virulence. PLoS Pathog. 13, e1006090 (2017).

18. Govender, V. S., Ramsugit, S. \& Pillay, M. Mycobacterium tuberculosis adhesins: potential biomarkers as anti-tuberculosis therapeutic and diagnostic targets. Microbiology 160, 1821-1831 (2014).

19. Connor, R. J., Kawaoka, Y., Webster, R. G. \& Paulson, J. C. Receptor specificity in human, avian, and equine H2 and H3 influenza virus isolates. Virology 205, 17-23 (1994).

20. Russell, R. J., Stevens, D. J., Haire, L. F., Gamblin, S. J. \& Skehel, J. J. Avian and human receptor binding by hemagglutinins of influenza A viruses. Glycoconj. J. 23, 85-92 (2006).

21. Walther, T. et al. Glycomic analysis of human respiratory tract tissues and correlation with influenza virus infection. PLoS Pathog. 9 , e1003223 (2013).

22. Ruhaak, L. R. et al. Differential N-Glycosylation Patterns in Lung Adenocarcinoma Tissue. J. Proteome Res. 14, 4538-4549 (2015).

23. Shinya, K. et al. Avian flu: influenza virus receptors in the human airway. Nat. 440, 435-436 (2006).

24. Byrd-Leotis, L. et al. Influenza binds phosphorylated glycans from human lung. Sci. Adv. 5, eaav2554 (2019).

25. Baenziger, J. U. \& Fiete, D. Structural determinants of concanavalin A specificity for oligosaccharides. J. Biol. Chem. 254, 2400-2407 (1979).

26. Shibuya, N. et al. Fractionation of sialylated oligosaccharides, glycopeptides, and glycoproteins on immobilized elderberry (Sambucus nigra L.) bark lectin. Arch. Biochem. Biophys. 254, 1-8 (1987).

27. Wang, W. C. \& Cummings, R. D. The immobilized leukoagglutinin from the seeds of Maackia amurensis binds with high affinity to complex-type Asn-linked oligosaccharides containing terminal sialic acid-linked alpha-2,3 to penultimate galactose residues. J. Biol. Chem. 263, 4576-4585 (1988).

28. Gao, C. et al. Unique Binding Specificities of Proteins toward Isomeric Asparagine-Linked Glycans. Cell Chem. Biol. 26, 535-547 (2019).

29. Kochibe, N. \& Furukawa, K. Purification and properties of a novel fucose-specific hemagglutinin of Aleuria aurantia. Biochem. 19, 2841-2846 (1980).

30. Matsumoto, I. \& Osawa, T. Purification and characterization of an anti-H(O) phytohemagglutinin of Ulex europeus. Biochim. Biophys. Acta 194, 180-189 (1969).

31. Quinn, J. M. \& Etzler, M. E. Isolation and characterization of a lectin from the roots of Dolichos biflorus. Arch. Biochem. Biophys. 258, 535-544 (1987).

32. Xia, B., Royall, J. A., Damera, G., Sachdev, G. P. \& Cummings, R. D. Altered O-glycosylation and sulfation of airway mucins associated with cystic fibrosis. Glycobiol. 15, 747-775 (2005).

33. Van Poucke, S. G., Nicholls, J. M., Nauwynck, H. J. \& Van Reeth, K. Replication of avian, human and swine influenza viruses in porcine respiratory explants and association with sialic acid distribution. Virol. J. 7, 38 (2010).

34. Couceiro, J. N., Paulson, J. C. \& Baum, L. G. Influenza virus strains selectively recognize sialyloligosaccharides on human respiratory epithelium; the role of the host cell in selection of hemagglutinin receptor specificity. Virus Res. 29, 155-165 (1993).

35. Nicholls, J. M., Bourne, A. J., Chen, H., Guan, Y. \& Peiris, J. S. Sialic acid receptor detection in the human respiratory tract: evidence for widespread distribution of potential binding sites for human and avian influenza viruses. Respir. Res. 8, 73 (2007).

36. Eriksson, P. et al. Characterization of avian influenza virus attachment patterns to human and pig tissues. Sci. Rep. 8, 12215 (2018).

37. Suzuki, Y. et al. Sialic acid species as a determinant of the host range of influenza A viruses. J. Virol. 74, 11825-11831 (2000).

38. Trebbien, R., Larsen, L. E. \& Viuff, B. M. Distribution of sialic acid receptors and influenza A virus of avian and swine origin in experimentally infected pigs. Virol. J. 8, 434 (2011).

39. Ning, Z. Y. et al. Detection of expression of influenza virus receptors in tissues of BALB/c mice by histochemistry. Vet. Res. Commun. 33, 895-903 (2009).

40. van Riel, D. et al. Human and avian influenza viruses target different cells in the lower respiratory tract of humans and other mammals. Am. J. Pathol. 171, 1215-1223 (2007).

41. Rogers, G. N. et al. Single amino acid substitutions in influenza haemagglutinin change receptor binding specificity. Nat. 304, 76-78 (1983).

42. Gupta, R., Matta, K. L. \& Neelamegham, S. A systematic analysis of acceptor specificity and reaction kinetics of five human $\alpha(2,3)$ sialyltransferases: Product inhibition studies illustrate reaction mechanism for ST3Gal-I. Biochem. Biophys. Res. Commun. 469, 606-612 (2016).

43. Noel, M. et al. Probing the CMP-Sialic Acid Donor Specificity of Two Human $\beta$-d-Galactoside Sialyltransferases (ST3Gal I and ST6Gal I) Selectively Acting on O- and N-Glycosylproteins. Chembiochem 18, 1251-1259 (2017).

44. Kuhn, B. et al. The structure of human $\alpha-2,6$-sialyltransferase reveals the binding mode of complex glycans. Acta Crystallogr. D. Biol. Crystallogr 69, 1826-1838 (2013).

45. Peng, W. et al. Recent H3N2 Viruses Have Evolved Specificity for Extended, Branched Human-type Receptors, Conferring Potential for Increased Avidity. Cell Host Microbe 21, 23-34 (2017). 
46. Gulati, S. et al. Human H3N2 Influenza Viruses Isolated from 1968 To 2012 Show Varying Preference for Receptor Substructures with No Apparent Consequences for Disease or Spread. PLoS One 8, e66325 (2013).

47. Chandrasekaran, A. et al. Glycan topology determines human adaptation of avian H5N1 virus hemagglutinin. Nat. Biotechnol. 26, 107-113 (2008)

48. Nemanichvili, N. et al. Fluorescent Trimeric Hemagglutinins Reveal Multivalent Receptor Binding Properties. J. Mol. Biol. 431, 842-856 (2019).

49. Sassaki, G. L. et al. Human $(\alpha 2 \rightarrow 6)$ and avian $(\alpha 2 \rightarrow 3)$ sialylated receptors of influenza A virus show distinct conformations and dynamics in solution. Biochem. 52, 7217-7230 (2013).

50. Byrd-Leotis, L. et al. Shotgun glycomics of pig lung identifies natural endogenous receptors for influenza viruses. Proc. Natl Acad. Sci. USA 111, E2241-2250 (2014).

51. Stevens, J. et al. Structure and receptor specificity of the hemagglutinin from an H5N1 influenza virus. Sci. 312, 404-410 (2006).

52. Bradley, K. C. et al. Comparison of the receptor binding properties of contemporary swine isolates and early human pandemic H1N1 isolates (Novel 2009 H1N1). Virology 413, 169-182 (2011).

53. Nycholat, C. M. et al. Recognition of sialylated poly-N-acetyllactosamine chains on $\mathrm{N}$ - and O-linked glycans by human and avian influenza A virus hemagglutinins. Angew. Chem. Int. Ed. Engl. 51, 4860-4863 (2012).

54. Chan, R. W. et al. Infection of swine ex vivo tissues with avian viruses including H7N9 and correlation with glycomic analysis. Influenza Other Respir. Viruses 7, 1269-1282 (2013).

55. Jia, N. et al. Glycomic characterization of respiratory tract tissues of ferrets: implications for its use in influenza virus infection studies. J. Biol. Chem. 289, 28489-28504 (2014).

56. Avril, T., North, S. J., Haslam, S. M., Willison, H. J. \& Crocker, P. R. Probing the cis interactions of the inhibitory receptor Siglec-7 with alpha2,8-disialylated ligands on natural killer cells and other leukocytes using glycan-specific antibodies and by analysis of alpha2,8-sialyltransferase gene expression. J. Leukoc. Biol. 80, 787-796 (2006).

57. Falco, M. et al. Identification and molecular cloning of p75/AIRM1, a novel member of the sialoadhesin family that functions as an inhibitory receptor in human natural killer cells. J. Exp. Med. 190, 793-802 (1999).

58. Marquardt, N. et al. Human lung natural killer cells are predominantly comprised of highly differentiated hypofunctional CD69. J. Allergy Clin. Immunol. 139, 1321-1330.e1324 (2017).

59. Freud, A. G., Mundy-Bosse, B. L., Yu, J. \& Caligiuri, M. A. The Broad Spectrum of Human Natural Killer Cell Diversity. Immun. 47, 820-833 (2017).

60. Bern, M. et al. Polylactosaminoglycan glycomics: enhancing the detection of high-molecular-weight N-glycans in matrix-assisted laser desorption ionization time-of-flight profiles by matched filtering. Mol. Cell Proteom. 12, 996-1004 (2013).

61. Ng, P. S. et al. Ferrets exclusively synthesize Neu5Ac and express naturally humanized influenza A virus receptors. Nat. Commun. 5, 5750 (2014).

62. Bradley, K. C. et al. Analysis of influenza virus hemagglutinin receptor binding mutants with limited receptor recognition properties and conditional replication characteristics. J. Virol. 85, 12387-12398 (2011).

63. North, S. J. et al. Mass spectrometric analysis of mutant mice. Methods Enzymol. 478, 27-77 (2010).

64. Strohalm, M., Hassman, M., Kosata, B. \& Kodícek, M. mMass data miner: an open source alternative for mass spectrometric data analysis. Rapid Commun. Mass. Spectrom. 22, 905-908 (2008).

65. Ceroni, A. et al. GlycoWorkbench: a tool for the computer-assisted annotation of mass spectra of glycans. J. Proteome Res. 7, $1650-1659$ (2008).

\section{Acknowledgements}

We are grateful to Dr. Jamie Heimburg-Molinaro, Dr. Mohui Wei and Dr. Mark Jones for valuable discussion and advice. We thank Dr. Lay-Hong Ang from Confocal Imaging Core at BIDMC for immunofluorescence staining of lung sections and Jennifer Wang from FAS Small Molecule Mass Spectrometry Facility at Harvard University for running GC-MS linkage analysis. The authors acknowledge support by the U.S. Department of Health and Human Services contract HHSN272201400004C (NIAID Centers of Excellence for Influenza Research and Surveillance) and NIH grant P41GM103694 to R.D.C.

\section{Author contributions}

D.A.S. and R.D.C. supervised, conceived and coordinated the study. N.J., L.B.L. and Y.M. performed the experiments. N.J., L.B.L., Y.M. and C.G. analyzed the data. A.N.W., J.L.W. and J.E.K. prepared the human lung sections. N.J., L.B.L., D.A.S. and R.D.C. wrote the manuscript. All authors contributed to review and editing of the manuscript.

\section{Competing interests}

The authors declare no competing interests.

Additional information

Supplementary information is available for this paper at https://doi.org/10.1038/s41598-020-62074-z.

Correspondence and requests for materials should be addressed to D.A.S. or R.D.C.

Reprints and permissions information is available at www.nature.com/reprints.

Publisher's note Springer Nature remains neutral with regard to jurisdictional claims in published maps and institutional affiliations.

(c) (i) Open Access This article is licensed under a Creative Commons Attribution 4.0 International License, which permits use, sharing, adaptation, distribution and reproduction in any medium or format, as long as you give appropriate credit to the original author(s) and the source, provide a link to the Creative Commons license, and indicate if changes were made. The images or other third party material in this article are included in the article's Creative Commons license, unless indicated otherwise in a credit line to the material. If material is not included in the article's Creative Commons license and your intended use is not permitted by statutory regulation or exceeds the permitted use, you will need to obtain permission directly from the copyright holder. To view a copy of this license, visit http://creativecommons.org/licenses/by/4.0/.

(C) The Author(s) 2020 